\title{
Wideband Waveform Design for Robust Target Detection
}

\author{
Ashkan Panahi*, Marie Ström*†, Mats Viberg* \\ * Signal Processing Group, Signals and Systems Department, Chalmers University, Gothenburg, Sweden \\ $\dagger$ Saab EDS, Gothenburg, Sweden \\ Email: \{ashkanp,marie.strom,viberg\}@chalmers.se
}

\begin{abstract}
Future radar systems are expected to use waveforms of a high bandwidth, where the main advantage is an improved range resolution. In this paper, a technique to design robust wideband waveforms for a Multiple-Input-Single-Output system is developed. The context is optimal detection of a single object with partially unknown parameters. The waveforms are robust in the sense that, for a single transmission, detection capability is maintained over an interval of time-delay and time-scaling (Doppler) parameters. A solution framework is derived, approximated, and formulated as an optimization by means of basis expansion. In terms of probabilities of detection and false alarm, numerical evaluation shows the efficiency of the proposed method when compared with a Linear Frequency Modulated signal and a Gaussian pulse.
\end{abstract}

\section{INTRODUCTION}

Signal processing techniques for radar systems have to a great extent focused on narrowband signals. At this time, signal generators are able to synthesize arbitrary signals with a bandwidth of the order of $\mathrm{GHz}[1]-[3]$. On one hand, this provides interesting new opportunities, e.g., a wideband signal achieves an increased range resolution compared to its narrowband counterpart [4]-[7]. On the other hand, the simplifying narrowband assumption, where velocity is approximated as a frequency shift, is not valid [7]. This does not only complicate the design, but also disqualifies traditional detection techniques, as estimation of time-delay and Doppler-shift can not be separated in time and frequency. It should also be considered that, for some applications, maintaining a low computational complexity is crucial, which is naturally against the original desire of super-resolution. Accordingly, this work is devoted to provide an adaptive detection scheme, which establishes an arbitrary trade-off between complexity and resolution.

The above concern is related to other contributions in the area of waveform design. For example, design of wideband ambiguity functions with narrow peaks for Orthogonal-Frequency-Division-Multiplexing signals is considered in [8], [9]. In [10] various techniques for designing narrowband or wideband waveforms are discussed. Interesting analysis of wideband radar systems from various perspectives are also found in [11]-[13]. However, 
these studies mostly focus on designing ambiguity functions with a narrow peak neglecting complexity. It is easy to see that applying these methods to the discussed topic leads to a large number of transmissions, due to lack of shift-invariance properties, as well as a large set of detection filters.

To combat complexity, this work proposes a waveform design method that maintains a necessary mainlobe width in the ambiguity function, thus decreasing the overall number of pulse transmissions and receive filters. Although this leads to detections of a restricted resolution, it is easy to adapt a secondary super-resolution detection procedure [14]-[16] based on the original estimates. This provides a highly flexible design with low complexity.

Here, focuses is on obtaining original estimates of a restricted resolution. This is carried out by considering relatively wide parameter ranges and designing corresponding waveforms, for which reliable detection, in the entire mesh, is ensured after filtering matched to a nominal value. This is not straightforward as wideband signals may lead to a focused ambiguity function, where off-grid targets are easily missed. A matched filter design is selected to ensure a good performance in presence of noise, assuming nominal parameter values. The main contribution of this work is to formulate this idea, and to provide waveforms that guarantee robust detection in a desired interval of target parameters. In what follows, a statistical framework for detecting a single target is developed, and for which expressions are simplified by approximation to obtain a tractable design.

\section{Problem Formulation}

Consider a bistatic radar system that, on the transmitter side, employs $M$ waveform generators each connected to an antenna element. The receiver side comprises one antenna element connected to a filter bank. Each generator samples a baseband signal composed of a set of $N$ basis functions $\psi_{m, n}(t)$, where $m$ and $n$ are the antenna and basis label, respectively. In other words,

$$
\tilde{x}_{m}(t)=\sum_{n=1}^{N} s_{m, n} \psi_{m, n}(t)
$$

where $\tilde{x}_{m}$ is the waveform at the $m$ th signal generator, and $s_{m, n}$ is a complex scalar coefficient. The received signal is a mixture of the reflected transmitted waveforms, and can be expressed, for a point target, as

$$
y(t)=\sigma_{t} \sum_{m=1}^{M} x_{m}\left(\mu\left(t-\tau_{m}(\phi)-\tau\right)\right)+n(t) .
$$

where $\sigma_{t}$ is the object's reflection coefficient, $\tau$ denotes the time-delay from the zero-phase sensor to the receiver, and $\mu$ is the time-scaling related to the velocity of an object [6], [7]. Furthermore, $\tau_{m}(\phi)$ is given by the inter-element spacing and the spatial direction, $\phi$, towards the object. This direction, azimuth and/or elevation, is assumed to be known. If this is not the case, a beamforming technique, see, e.g., [17]-[19], is necessary. In (2), $x_{m}(t)=\tilde{x}_{m}(t) e^{j \omega_{c} t}$ is centered around the system's carrier frequency $f_{c}=\omega_{c} / 2 \pi$ and $n(t)$ is a white Gaussian noise.

At the receiver side the down-converted signal, $\tilde{y}(t)=y(t) e^{-j \omega_{c} t}$, is passed through a filter bank, i.e.,

$$
r\left(\tau, \tau^{\prime}, \mu, \mu^{\prime}\right)=\int h^{*}\left(t ; \tau^{\prime}, \mu^{\prime}\right) \tilde{y}(t) d t .
$$

Here, $(\cdot)^{*}$ denotes the complex conjugate. If a so-called matched filter structure [20], [21] is employed, the correlating filters, $h\left(t ; \tau^{\prime}, \mu^{\prime}\right)$, are equal to the received signal calculated from their corresponding transmission model. 
To ease calculations, assume that the basis kernels, $\psi_{m, n}(t)$, are Gaussian. This particular choice of functions results in that (3) can be analytically calculated to

$$
\begin{aligned}
& r\left(\tau_{0}, \mu, \mu^{\prime}\right)=\sum_{\substack{m, m^{\prime} \in \mathcal{M} \\
n, n^{\prime} \in \mathcal{N}}} \frac{\sigma_{t} \sqrt{\mu \mu^{\prime}} s_{m, n} s_{m^{\prime}, n^{\prime}} e^{-j \omega_{c} \mu^{\prime}\left(\tau_{0}+\tau_{m, m^{\prime}}\right)}}{\sqrt{2 \pi\left(\mu^{2} \sigma_{m^{\prime}, n^{\prime}}^{2}+\mu^{\prime 2} \sigma_{m, n}^{2}\right)}} .
\end{aligned}
$$

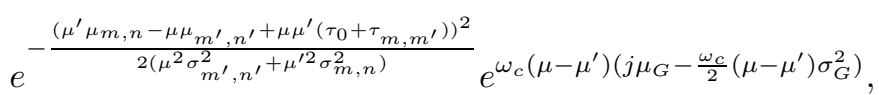

where $\mathcal{M}=\{1 \ldots M\}, \mathcal{N}=\{1 \ldots N\}, \sigma_{m, n}^{2}$ and $\mu_{m, n}$ correspond to the variance and the mean of the $n$th basis kernel sampled by the $m$ th signal generator, respectively, $\tau_{0}=\tau-\tau^{\prime}, \tau_{m, m^{\prime}}=\tau_{m}(\phi)-\tau_{m^{\prime}}(\phi)$, and

$$
\begin{aligned}
\mu_{G} & =\frac{\mu \mu_{m, n} \sigma_{m^{\prime}, n^{\prime}}^{2}+\mu^{\prime}\left(\mu_{m^{\prime}, n^{\prime}}-\mu^{\prime}\left(\tau_{0}+\tau_{m, m^{\prime}}\right)\right) \sigma_{m, n}^{2}}{\mu^{2} \sigma_{m^{\prime}, n^{\prime}}^{2}+\mu^{\prime 2} \sigma_{m, n}^{2}} \\
\sigma_{G}^{2} & =\frac{\sigma_{m, n}^{2} \sigma_{m^{\prime}, n^{\prime}}^{2}}{\mu^{2} \sigma_{m^{\prime}, n^{\prime}}^{2}+\mu^{\prime 2} \sigma_{m, n}^{2}} .
\end{aligned}
$$

The expression in (4) is equivalently written in a matrix form as

$$
r\left(\tau_{0}, \mu, \mu^{\prime}\right)=\sigma_{t} \mathbf{s}^{H} \mathbf{R}\left(\tau_{0}, \mu, \mu^{\prime}\right) \mathbf{s}
$$

where $\mathbf{s}=\left[s_{1,1} \ldots s_{N, 1}, s_{1,2} \ldots s_{N, 2} \ldots s_{N, M}\right]^{T}$ and $\mathbf{R}\left(\tau_{0}, \mu, \mu^{\prime}\right)$ contains the entries

$$
\begin{aligned}
& R_{m, m^{\prime}, n, n^{\prime}}\left(\tau_{0}, \mu, \mu^{\prime}\right)=\frac{\sigma_{t} \sqrt{\mu \mu^{\prime}} e^{-j \omega_{c} \mu^{\prime}\left(\tau_{0}+\tau_{m, m^{\prime}}\right)}}{\sqrt{2 \pi\left(\mu^{2} \sigma_{m^{\prime}, n^{\prime}}^{2}+\mu^{\prime 2} \sigma_{m, n}^{2}\right)}} \cdot \\
& e^{-\frac{\left.\left(\mu^{\prime} \mu_{m, n}-\mu \mu_{m^{\prime}, n^{\prime}+\mu \mu^{\prime}\left(\tau_{0}+\tau\right.}+m^{\prime}\right)\right)^{2}}{2\left(\mu^{2} \sigma_{m^{\prime}, n^{\prime}}^{2}+\mu^{\prime 2} \sigma_{m, n}^{2}\right)}} e^{\omega_{c}\left(\mu-\mu^{\prime}\right)\left(j \mu_{G}-\frac{\omega_{c}}{2}\left(\mu-\mu^{\prime}\right) \sigma_{T}^{2}\right)},
\end{aligned}
$$

in a proper order.

\section{A. Robust Waveform Design Based on Statistical Performance}

Target detection is formulated as a statistical problem as follows. Take a region $\mathcal{S}$ of the parameter pairs $(\mu, \tau)$ in which a good detection performance is desired. The detection is based on the output $r$ from a filter matched to a nominal point $\left(\tau^{\prime}, \mu^{\prime}\right) \in \mathcal{S}$. A matched filter [20], [21] ensures maximal detection performance when the true parameters are nominal in the presence of white noise.

The idea is to develop a simple decision rule for $r$, which identifies whether a target is present in $\mathcal{S}$ or not. The problem can be formulated as a composite hypothesis testing, where $\mathcal{H}_{0}$ denotes the hypothesis that no source is present, in which $r$ is generated by a white Gaussian noise processes, and $\mathcal{H}_{1}$ denotes the composite hypothesis of source existence. Then, the likelihood functions under the different hypotheses are

$$
\begin{aligned}
& \mathcal{H}_{0}: r \sim \mathcal{N}\left(0, \sigma^{2} \mathbf{s}^{H} \mathbf{R}_{0} \mathbf{s}\right) \\
& \mathcal{H}_{1}: r \sim \mathcal{N}\left(\sigma_{t} \mathbf{s}^{H} \mathbf{R}\left(\tau_{0}, \mu, \mu^{\prime}\right) \mathbf{s}, \sigma^{2} \mathbf{s}^{H} \mathbf{R}_{0} \mathbf{s}\right),
\end{aligned}
$$

where $\mathbf{R}_{0}=\mathbf{R}\left(\tau_{0}=0, \mu^{\prime}, \mu^{\prime}\right)$ and $\sigma^{2}$ denotes the noise power. The pair $\left(\tau_{0}, \mu\right)$ denotes the unknown true parameters. Later, we simply refer to it as $\theta$. Let us consider the Generalized Likelihood Ratio Test (GLRT) [22], which can be written as

$$
\min _{\theta, \sigma_{t}}\left|r-\sigma_{t} \mathbf{s}^{H} \mathbf{R}(\theta) \mathbf{s}\right|^{2}+\gamma \gtrless|r|^{2},
$$


where the argument $\mu^{\prime}$ is dropped as its value is assumed to be fixed, and $\gamma$ is a threshold. As $\sigma_{t}$ is free the minimization on the left hand side gives zero. Thus, the GLRT simplifies to

$$
\gamma \gtrless|r|,
$$

which is a simple power thresholding scheme.

For this detector probabilities of detection, $P_{D}$, and false alarm, $P_{F A}$, can be calculated to

$$
\begin{aligned}
& P_{D}\left(\theta, \sigma_{t}, \gamma\right)=\frac{1}{\pi \mathbf{s}^{H} \mathbf{R}_{0} \mathbf{s} \sigma^{2}} \int_{|r|>\gamma} e^{-\frac{\left|r-\sigma_{t} \mathbf{s}^{H} \mathbf{R}(\theta) \mathbf{s}\right|^{2}}{\mathbf{s}^{H} \mathbf{R}_{0} \mathbf{s} \sigma^{2}}} \mathrm{~d} \nu(r) \\
& P_{F A}=\frac{1}{\pi \mathbf{s}^{H} \mathbf{R}_{0} \mathbf{s} \sigma^{2}} \int_{|r|>\gamma} e^{-\frac{|r|^{2}}{\mathbf{s}^{H} \mathbf{R}_{0} \mathbf{s} \sigma^{2}}} \mathrm{~d} \nu(r),
\end{aligned}
$$

where $\nu($.$) denotes the Lebesgue measure on the complex plane of r$.

To optimize the waveform, consider the worst detection performance $P_{D}\left(\theta, \sigma_{t}, \gamma\right)$ over all scenarios defined by $\left(\theta, \sigma_{t}\right)$, where $P_{F A}=\alpha$ is fixed. One may define the best design as the one maximizing the worst detection. Unfortunately, direct calculation shows that this approach fails in the current occasion as the worst detection performance is independent of the choice of waveform. To overcome this, denote by $P_{D \text {,worst }}(\alpha)$, or simply $P_{D \text {,worst }}$, the worst detection performance. Then, define an optimal design as follows:

Definition 1: A design $\mathbf{s}$ is optimal for a given value of $\alpha$ if for sufficiently small values of $\epsilon$, the set $\mathcal{S}_{\epsilon}$ of $\epsilon$-worse scenarios, defined by

$$
\mathcal{S}_{\epsilon}=\left\{\left(\theta, \sigma_{t}\right)|| P_{D}\left(\theta, \sigma_{t}\right)<P_{D, \text { worst }}+\epsilon\right\}
$$

has a minimal area (Lebesgue measure) in any compact region.

Clearly, this gives a design, where it is least likely to encounter a low performance, although not impossible. The following theorem provides a practical method to realize this design.

Theorem 1: The optimal design in the sense of Definition 1 is a solution to the following optimization

$$
\begin{array}{ll}
\underset{\mathbf{s}}{\max } \min _{\theta} & \left|\mathbf{s}^{H} \mathbf{R}(\theta) \mathbf{s}\right| \\
\text { s.t. } & \mathbf{s}^{H} \mathbf{R}_{0} \mathbf{s}=1 .
\end{array}
$$

The reader may notice that (12) could be directly introduced and intuitively validated. It is simple to verify that $\left|\mathbf{s}^{H} \mathbf{R}(\theta) \mathbf{s}\right|$ and $\mathbf{s}^{H} \mathbf{R}_{0} \mathbf{s}$ are the share of signal and noise in the filter output energy over $\mathcal{S}$, respectively. Thus, (12) promotes a uniformly high signal-output energy. However, the above calculations tie (12) to a statistically sound detection approach.

Further, $\mathbf{R}_{0}$ is positive semi-definite, and the term $\mathbf{s}^{H} \mathbf{R}_{0} \mathbf{s}$ characterizes the energy of the signal, which is typical for a matched filter design. Thus, no extra care about the transmit energy is considered. Clearly, (12) guarantees high detection rate, but it does not consider false detection due to coupling between the filter and out-of-box sources. However, it is expected that the finite energy constraint automatically enforces low sidelobe energy. We later argue on the validity of this assumption by analyzing numerical results. 


\section{Proposed Method For Solving (12)}

It is difficult to exactly solve (12), as $\mathbf{R}(\theta)$ is in general not Hermitian. One approximate solution is to consider the inner optimization over a finite number of grid points, $\theta_{1}, \theta_{2}, \ldots, \theta_{l}$. Then, (12) can be written as

$$
\begin{array}{ll}
\max _{\mathbf{s}} \min _{\lambda_{1}, \lambda_{2}, \ldots, \lambda_{l}} & \sum_{k}\left|\mathbf{s}^{H} \mathbf{R}\left(\theta_{k}\right) \mathbf{s}\right| \lambda_{k} \\
\text { s.t. } & \mathbf{s}^{H} \mathbf{R}_{0} \mathbf{s}=1, \quad \sum_{k} \lambda_{k}=1,
\end{array}
$$

where $\lambda_{k}$ is a positive number. By changing the order of minimization and maximization in (13) we obtain the following suboptimal design, which is simpler to solve [23].

$$
\begin{array}{ll}
\min _{\lambda_{1}, \lambda_{2}, \ldots, \lambda_{l}} \max _{\mathbf{s}} & \sum_{k}\left|\mathbf{s}^{H} \mathbf{R}\left(\theta_{k}\right) \mathbf{s}\right| \lambda_{k} \\
\text { s.t. } & \mathbf{s}^{H} \mathbf{R}_{0} \mathbf{s}=1, \quad \sum_{k} \lambda_{k}=1 .
\end{array}
$$

As opposed to the uniformly optimal design in the original order, the change of order results in an optimization considering average performance weighted by $\left\{\lambda_{k}\right\}$. However, as our simulation results indicate, the latter average design also leads to a remarkably good detection performance. Now, the inner optimization in (14) is simplified as follows. Consider the eigenvalue decomposition of $\mathbf{R}_{0}$, i.e.,

$$
\mathbf{R}_{0}=\mathbf{U} \Sigma \mathbf{U}^{H}=\mathbf{U}_{0} \Sigma_{0} \mathbf{U}_{0}^{H},
$$

where $\Sigma_{0}$ and $\mathbf{U}_{0}$ are the nonzero blocks of $\Sigma$ and its corresponding columns of $\mathbf{U}$, respectively. Let $\mathbf{u}=\boldsymbol{\Sigma}_{\mathbf{0}}{ }^{\frac{1}{2}} \mathbf{U}_{\mathbf{0}}{ }^{H} \mathbf{s}$, which implies that any vector $\mathbf{s}$ is uniquely decomposed in terms of its corresponding $\mathbf{u}$ as

$$
\mathbf{s}=\mathbf{U}_{\mathbf{0}} \boldsymbol{\Sigma}_{\mathbf{0}}^{-\frac{1}{2}} \mathbf{u}+\mathbf{U}_{\mathbf{1}} \mathbf{p}
$$

where $\mathbf{p}$ is a suitable vector and $\mathbf{U}_{\mathbf{1}}$ spans the null space of $R_{0}$. Note that, any vector $\mathbf{s}$ with $\mathbf{s}^{H} \mathbf{R}_{0} \mathbf{s}=0$ corresponds to zero-energy, which leads to a zero output-signal. This clearly means that $\mathbf{R}(\theta) \mathbf{U}_{1}=0$ for every $\theta$. Thus, the term $U_{1}$ does not have any effect on the waveform design, and the inner optimization in (14) can be expressed as

$$
\begin{array}{ll}
\max _{\mathbf{u}} & \left.\sum_{k} \lambda_{k} \mid \mathbf{u}^{H} \tilde{\mathbf{R}}\left(\theta_{k}\right) \mathbf{u}\right) \mid \\
\text { s.t. } & \|\mathbf{u}\|_{2}^{2}=1,
\end{array}
$$

where $\tilde{\mathbf{R}}\left(\theta_{k}\right)=\boldsymbol{\Sigma}_{0}^{-1 / 2} \mathbf{U}_{\mathbf{0}}{ }^{H} \mathbf{R}\left(\theta_{k}\right) \mathbf{U}_{\mathbf{0}} \boldsymbol{\Sigma}_{\mathbf{0}}{ }^{-\frac{1}{2}}$.

To solve (17) we propose the following efficient scheme. First, note that, $|\alpha|=\max _{\phi} \Re\left(e^{-j \phi} \alpha\right)$. Thus, (17) is equivalently written as

$$
\begin{aligned}
& \max _{\mathbf{u}, \phi_{1}, \phi_{2}, \ldots, \phi_{l}} \sum_{k} \lambda_{k} \Re\left(e^{-j \phi_{k}} \mathbf{u}^{H} \tilde{\mathbf{R}}\left(\theta_{k}\right) \mathbf{u}\right) \\
& =\max _{\mathbf{u}, \phi_{1}, \phi_{2}, \ldots, \phi_{l}} \mathbf{u}^{H} \mathbf{M}\left(\phi_{1}, \phi_{2}, \ldots, \phi_{l}\right) \mathbf{u},
\end{aligned}
$$

where

$$
\mathbf{M}\left(\phi_{1}, \phi_{2}, \ldots, \phi_{l}\right)=\sum_{k} \lambda_{k}\left(e^{-j \phi_{k}} \tilde{\mathbf{R}}\left(\theta_{k}\right)+e^{j \phi_{k}} \tilde{\mathbf{R}}^{H}\left(\theta_{k}\right)\right)
$$


As the optimization is performed over all unit vectors $\mathbf{u}$, the solution for a fixed choice of $\phi_{1}, \ldots, \phi_{l}$ is the eigenvector of $\mathbf{M}$ corresponding to the largest eigenvalue, which we denote by $\mathbf{u}_{m}(\mathbf{M})$ and $\lambda_{m}(\mathbf{M})$, respectively. Accordingly, we propose the following cyclic solution of the inner optimization.

1) Start from an arbitrary choice of $\phi_{k}^{0}$ and set $r=1$.

2) Get $\mathbf{M}^{r-1}=\mathbf{M}\left(\phi_{1}^{r-1}, \ldots, \phi_{n}^{r-1}\right)$ and set $\mathbf{u}^{r}=\mathbf{u}_{m}\left(\mathbf{M}^{r-1}\right)$ by calculating $\lambda_{m}\left(\mathbf{M}\left(\phi^{r-1}\right)\right)$.

3) Evaluate $\phi_{k}^{n}$ as the argument of the complex number $\left(\mathbf{u}^{r}\right)^{H} \tilde{\mathbf{R}}\left(\theta_{k}\right) \mathbf{u}^{r}$, update $r$ to $r+1$ and go to step 2 .

Step 2 and step 3 increase the cost of (18) with respect to $\mathbf{u}$ and $\phi$. Thus, the cost monotonically increases, which guarantees convergence.

Once a solution, say $\overline{\mathbf{u}}$, for the inner optimization and given values of $\lambda_{k}$ is obtained, a local optimization technique such as steepest descent is performed to update $\lambda_{k}$ for the outer minimization. Although, the gradient, $\nabla F$, of the cost $F=F\left(\lambda_{1}, \ldots, \lambda_{l}\right)$ at a given point is simple to compute, applying a steepest decent technique is generally difficult. However, the number of grid points, $l$, in (13) may be significantly low as a result of sufficient correlation between conceivable return signals. In fact, we employ only a pair of properly selected grid points, typically the corner points of a parameter box, from which the outer optimization can be substantially simplified to

$$
\min _{0 \leq \lambda \leq 1} F(\lambda, 1-\lambda)
$$

and a resulting 1-dimensional optimization is either carried out by a grid search or a bisection method [24].

\section{Numerical Validation}

For different choices of regions in which a robust performance is desired, the efficiency of the proposed algorithm is presented in terms of average and minimum correlation as well as Receiver Operating Characteristic (ROC) [25] curves. To examine the method, we calculate its efficiency by Monte-Carlo simulation in selected scenarios. The number of waveform generators is $M=3$, for each $N=30$ basis functions are generated. The system has a bandwidth-time product of 200 , the nominal value of the time-scaling is $\mu^{\prime}=0.94$, and the target's reflection coefficient is set to $\sigma_{t}=1$.

The Gaussian basis functions are generated with mean values, $\mu_{k}$, uniformly located over the pulse duration, and their standard deviations are selected randomly within an interval $\left[\sigma_{\min , k} \sigma_{\max , k}\right]$, where $\sigma_{\max , k}$ is such that the effective interval between $\mu_{k} \pm 3 \sigma_{k}$ is ensured to entirely lie within the pulse time, and $\sigma_{\min , k}$ restricts the highest effective frequency component.

The region in which the performance is evaluated is chosen as a box, which varies in size. The smallest box is within the confines of $\mu \in\left[\mu_{0}-\epsilon_{\mu}, \mu_{0}+\epsilon_{\mu}\right]$ and $\tau \in\left[-\epsilon_{\tau}, \epsilon_{\tau}\right]$, where $\epsilon_{\mu}$ and $\epsilon_{\tau}$ are determined with respect to twice the system's resolution limits. In order to investigate the effect of varying uncertainty, the box size increases with a factor $\beta$ to $\epsilon_{\mu} / \beta$ and $\epsilon_{\tau} / \beta$, where $\beta=[0.9, \ldots, 0.1]$.

The minimum and average in-box correlation, $\left|\mathbf{s}^{H} \mathbf{R}(\theta) \mathbf{s}\right|$, are presented in Table I and Table II in which the results are averaged over 100 random choices of basis functions. The correlation properties are evaluated over a dense grid and then taking average or minimum, respectively. The outcome is compared with the cases where a 
Linear Frequency Modulated (LFM) pulse and a single Gaussian pulse is transmitted from each signal generator, with the same bandwidth-time product. It should be noted that the LFM pulse provides a high resolution, and is not expected to give a robust performance.

TABLE I: Average correlation properties of the designed waveforms.

\begin{tabular}{lccccc} 
Algorithm & $\beta=1$ & $\beta=0.8$ & $\beta=0.6$ & $\beta=0.4$ & $\beta=0.2$ \\
\hline Proposed alg. & 0.99 & 0.98 & 0.97 & 0.94 & 0.65 \\
Gaussian & 0.97 & 0.93 & 0.88 & 0.80 & 0.60 \\
LFM & 0.30 & 0.26 & 0.21 & 0.15 & 0.09
\end{tabular}

TABLE II: Minimum correlation properties of the designed waveforms.

\begin{tabular}{lccccc} 
Algorithm & $\beta=1$ & $\beta=0.8$ & $\beta=0.6$ & $\beta=0.4$ & $\beta=0.2$ \\
\hline Proposed alg. & 0.98 & 0.96 & 0.93 & 0.85 & 0.41 \\
Gaussian & 0.88 & 0.83 & 0.70 & 0.42 & 0.1 \\
LFM & 0.0039 & 0.0018 & 0.0018 & 0.0018 & 0.0016
\end{tabular}

Figure 1 shows the ROC curves. These curves are calculated from the statistical formulation through a Monte Carlo simulation using the estimator in (9) with $10^{6}$ noise realizations, and a Signal-to-Noise Ratio (SNR) of $10 \mathrm{~dB}$. The curves are also averaged over 10 independent draws of basis functions. The Figure shows the characteristics when $\beta=[1,0.8,0.6,0.4,0.2]$, for a varying threshold $\gamma=[0,0.05, \ldots, 4]$.

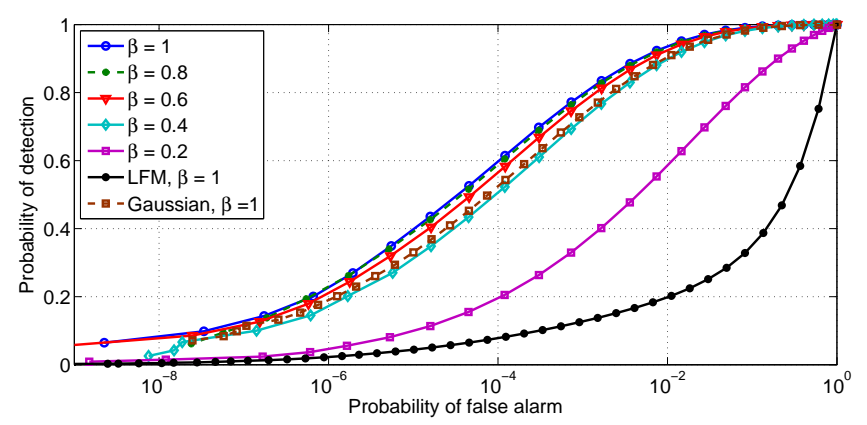

Fig. 1: ROC curves when the SNR is $10 \mathrm{~dB}$ for regions specified by $\beta=[1,0.8,0.6,0.4,0.2]$.

The curve corresponding to $\beta=1$ illustrates the performance in which the smallest box is selected. This curve is the nominal performance. As seen, the performance decreases when expanding the region. However, it exhibits a robust behavior for relatively large boxes.

The last part of the numerical results presents how an out-of-region source will affect the ROC curve. This kind of source increases the probability of false alarm if its return is highly correlated with the matched filter for the region of interest. The position of the source is randomly generated outside the interval of $\mu$ and $\tau$. Results shown in 
Figure 2 illustrates ROC curves when the out-of-region source has a reflection coefficient of $\sigma_{o s}=1$. The outcome when $\sigma_{o s}=-1$ coincides with $\sigma_{o s}=1$, and is therefore omitted in the Figure. The result is compared with the corresponding ROC curve when no such source exists for waveforms optimized with $\beta=0.6$.

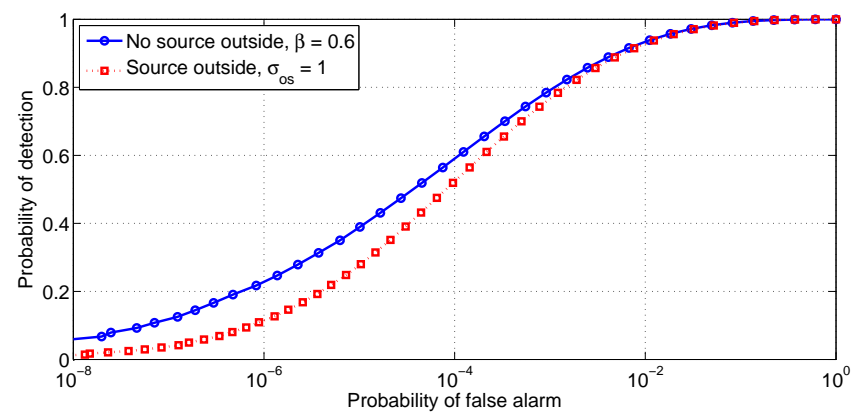

Fig. 2: ROC curve when an out-of-region source with $\sigma_{o s}=1$ is present. The SNR is $10 \mathrm{~dB}$ and $\beta=0.6$.

\section{CONCLUSIONS}

For a wideband radar, we considered a robust technique for waveform design within relatively wide parameter ranges. The method was developed from a statistical framework for detecting a single target, and simplified by approximation to obtain a tractable design. Being robust implies that the waveforms are not designed to provide good resolution properties. Therefore, a next step is to apply a super-resolution technique to the restricted area obtained from this first stage.

The correlating filters at the receiver side were selected to have a matched filter structure. We remark that, a different kind of filter design, e.g., [26]-[28] might result in better performance, which is a topic for future investigation.

As shown by numerical validation, the method ensured reliable detection in the desired range of target parameters. The probabilities of detection and false alarm are illustrated with ROC curves, which showed a small loss of performance when increasing the desired region of reliable detection up to a certain size. The outcome was compared with two conventional transmit signal designs and showed an increased performance for the investigated problem. Note that, LFM signals have good resolution properties, which makes them unsuitable for the discussed application. It was also seen that in presence of an out-of-region source the detection properties are only slightly affected, which implied that the design promoted a low sidelobe energy.

\section{REFERENCES}

[1] J. Han and C. Nguyen, "A new ultra-wideband, ultra-short monocycle pulse generator with reduced ringing," Microwave and Wireless Components Letters, IEEE, vol. 12, no. 6, pp. 206-208, June 2002.

[2] Y. Zhu, J. Zuegel, J. Marciante, and H. Wu, "Distributed waveform generator: A new circuit technique for ultra-wideband pulse generation, shaping and modulation," Solid-State Circuits, IEEE Journal of, vol. 44, no. 3, pp. 808-823, March 2009.

[3] D. Wentzloff and A. Chandrakasan, "Gaussian pulse generators for subbanded ultra-wideband transmitters," Microwave Theory and Techniques, IEEE Transactions on, vol. 54, no. 4, pp. 1647-1655, June 2006. 
[4] J. D. Taylor, Introduction to ultra-wideband radar systems. CRC press, 1994.

[5] H. Khan, W. Malik, D. Edwards, and C. Stevens, "Ultra wideband multiple-input multiple-output radar," in Radar Conference, 2005 IEEE International, May 2005, pp. 900-904.

[6] M. Hussain, "Ultra-wideband impulse radar- an overview of the principles," Aerospace and Electronic Systems Magazine, IEEE, vol. 13, no. 9, pp. 9-14, 1998.

[7] L. Weiss, "Wavelets and wideband correlation processing," Signal Processing Magazine, IEEE, vol. 11, no. 1, pp. 13-32, 1994.

[8] S. Sen and A. Nehorai, "Adaptive design of ofdm radar signal with improved wideband ambiguity function," Signal Processing, IEEE Transactions on, vol. 58, no. 2, pp. 928-933, 2010.

[9] _ _ "Target detection in clutter using adaptive ofdm radar," Signal Processing Letters, IEEE, vol. 16, no. 7, pp. 592-595, July 2009.

[10] H. He, J. Li, and P. Stoica, Waveform design for active sensing systems: a computational approach. Cambridge University Press, 2012.

[11] D. Lush and D. Hudson, "Ambiguity function analysis of wideband radars," in Radar Conference, 1991., Proceedings of the 1991 IEEE National, 1991, pp. 16-20.

[12] B. Yazici and G. Xie, "Wideband extended range-doppler imaging and waveform design in the presence of clutter and noise," Information Theory, IEEE Transactions on, vol. 52, no. 10, pp. 4563-4580, 2006.

[13] G. S. Antonio and D. Fuhrmann, "Beampattern synthesis for wideband mimo radar systems," in 1st IEEE Int. workshop on Comp. Advances in Multi-Sensor Adaptive process., Puerto Vallarta, Mexico, December 2005, pp. 105-108.

[14] S. Borison, S. B. Bowling, and K. M. Cuomo, "Super-resolution methods for wideband radar," 1992.

[15] K. M. Cuomo, J. E. Pion, and J. T. Mayhan, "Ultrawide-band coherent processing," Antennas and Propagation, IEEE Transactions on, vol. 47, no. 6, pp. 1094-1107, 1999.

[16] J. Moore and H. Ling, "Super-resolved time-frequency analysis of wideband backscattered data," Antennas and Propagation, IEEE Transactions on, vol. 43, no. 6, pp. 623-626, 1995.

[17] A. Gershman, "Robust adaptive beamforming in sensor arrays," AEU International Journal of Electronics and Communications, vol. 53, no. 6, pp. 305-314, 1999.

[18] J. Li and P. Stoica, Robust adaptive beamforming. Wiley Online Library, 2006.

[19] S. A. Vorobyov, A. B. Gershman, and Z.-Q. Luo, "Robust adaptive beamforming using worst-case performance optimization: A solution to the signal mismatch problem," Signal Processing, IEEE Transactions on, vol. 51, no. 2, pp. 313-324, 2003.

[20] M. Skolnik, Radar Handbook. New York, NY: The McGraw-Hill Companies, 1976.

[21] _ Introduction to Radar Systems. New York, NY: The McGraw-Hill Companies, 1981.

[22] S. M. Kay, "Fundamentals of statistical signal processing: Detection theory, vol. 2," 1998.

[23] M. S. Bazaraa, H. D. Sherali, and C. M. Shetty, Nonlinear programming: theory and algorithms. John Wiley \& Sons, 2013.

[24] R. Fletcher, Practical methods of optimization. John Wiley \& Sons, 2013.

[25] M. A. Richards, Fundamentals of radar signal processing. Tata McGraw-Hill Education, 2005.

[26] M. H. Ackroyd and F. Ghani, “Optimum mismatched filters for sidelobe suppression,” Aerospace and Electronic Systems, IEEE Transactions on, vol. AES-9, no. 2, pp. 214-218, March 1973.

[27] P. Stoica, J. Li, and M. Xue, "On binary probing signals and instrumental variables receivers for radar," Information Theory, IEEE Transactions on, vol. 54, no. 8, pp. 3820-3825, Aug 2008.

[28] — "Transmit codes and receive filters for radar," Signal Processing Magazine, IEEE, vol. 25, no. 6, pp. 94-109, November 2008. 